\title{
Cytotoxic and Apoptotic EfFects of Caffeic ACID AND CAFFeOYl MaLiC ACID ON BRAIN GLIOBLASTOMA (U87- MG)
}

\author{
Nurdan Sena Değirmenci ${ }^{1}$, Zeynep Ülker Akal ${ }^{1 *}$, Lokman Alpsoy $^{2}$ \\ ${ }^{1}$ Department of Biology, ${ }^{2}$ Medical Biology, Fatih University, Büyükçekmece, Istanbul-34500, Turkey \\ *E-mail: zulker@fatih.edu.tr \\ Tel.: +9021286633 00-2041; Fax: +902128663402.
}

Received: Sep 28, 2015 / Revised: Jan 15, 2016 / Accepted: Jan 16, 2016

\begin{abstract}
Urtica dioica is a kind of plant which is a member of Urticaceae family. Alternative medicines have attracted much attention as potential therapeutic agents in the prevention of cancer. As a medicinal plant, Urtica dioica has been used traditionally as a cancer treatment in place of chemotherapy. Caffeic acid and caffeoyl malic acid are phenolic compounds found in Urtica dioica. They have biological protective effects on human health such as diabetes, cancer and inflammation. The present study determines the cytotoxic and apoptotic effects of different concentrations $(1,5,10,50 \mu \mathrm{M})$ of caffeic acid (CA) and caffeoyl malic acid (CMA) on glioblastoma cell line (U87- MG). Cytotoxic activity studied by using xCELligence (real-time cell counter) and lactate dehydrogenease (LDH) assay systems against U87- MG. Antiproliferative activity also determined by WST-1 test. And lastly TUNEL assay used to show apoptosis induced by CA and CMA on U87- MG. All experiments were run for 48h. Our results show that CA especially in $50 \mu \mathrm{M}$ concentration has apoptotic and anti-proliferative effects on U87 cell line at 48th hour. 1, 5, $10 \mu \mathrm{M}$ concentrations of CMA decreased cell number according to the results of XCELligence and WST-1 at 48th hour. Especially CMA IC50 values of 20 $\mu \mathrm{M}$ also has more apoptotic and anti-proliferative effects on $U 87$ cell line. On the basis of our studies, CMA has anti-proliferative and apoptotic effect on $U 87$ cell lines dependent dose and time manner. As a conclusion we can say that CMA may be used to treat glioblostoma cancer cell types alternatively instead of high toxic chemotherapeutic drugs.
\end{abstract}

Key words: Urtica dioica, Urticaceae, Anticancer, Phenolic compounds, Medicinal plants.

\section{INTRODUCTION}

Urtica dioica, commonly called stinging nettle is a species of the Urticaceae which is a part of the larger group Order Urticales. U. dioica is a perennial herb with rhizomes, stolons and abundant stinging trichomes. The plant is spreading to various regions of the world. It prefers nutrient riches and lighted places. It is grown especially in the Black Sea region and other parts of Turkey. It is also cultivated in North and South Africa, China, India, Australia, New Zealand and North and South America and is native throughout Europe The roots, seeds and leaves of $U$. dioica have been used in folk medicine worldwide. It has beneficial effects on health such as nasal and menstrual haemorrhage, diabetes, rheumatism, eczema, anaemia hair loss, hypotensive, antiinflammatory effects, prostatic hyperplasia, diuretic and immunomodulatory activity. In addition some extracts or components of $U$. dioica have been using for cancer patients (Farzami et al 2003; Guil-Guerrero et al 2003). The leaves, seeds and roots of $U$. dioica contain different chemical compounds. It consists of glucopyranosides, glycoprotein, protein, flavonol glycosides, carotenoids as well as biologically active compounds, such as caffeic acid, caffeoyl 